\title{
Structure determination of nanocrystals
}

\author{
Junliang Sun \\ Peking University, Beijing, China, People's Republic of; \\ junliang.sun@pku.edu.cn
}

Structure determination of nano-size crystals is always a challenging problem. For quite lots of materials, it is very difficult to synthesize large/good enough crystals for single crystal X-ray diffraction studies. Powder X-ray diffraction (PXRD) is the major method for their atomic structure determination, PXRD is a quite mature technique and lots of powder structures were solved. However, for complicated structures with huge unit cell dimensions or those with crystal sizes smaller than 100nm, it is quite often to have severe peak overlapping problems, which makes it extremely difficult to solve the structure from PXRD alone. Electrons which interact with matter much stronger than X-ray can produce single-crystal-like diffraction from nano-crystalline materials, which makes it possible to collect single-crystal-like diffraction data.

The 3D electron diffraction technique can be used for collecting 3D electron diffraction data. Compared with traditional electron diffraction methods, this technique gives lower dynamical effects and much higher data completeness. Using the intensities abstracted from the data, complicated structures can be directly solved using the similar methods as single-crystal X-ray diffraction. Combining it with other techniques, such as PXRD or even SXRD, more complicated structures can be solved.

Keywords: nanomaterials, structure determination, 3D electron diffraction 